\title{
HSDPA Throughput Performance With Limited Number of Signal Paths
}

\author{
Juan Diego Sánchez-Heredia, Paul Hallbjörner, Thomas Bolin, and Antonio M. Martínez-González
}

\begin{abstract}
Over-the-air characterization of wireless devices is performed using specific channel models. Channel models define the distribution of the incident signals over angle and polarization, but always assuming a large number of signal paths. However, in some real cases, the number of signal paths can be limited. This letter studies the effect of the number of signal paths on the high-speed downlink packet access (HSDPA) throughput, using a commercial phone. Differences in performance are found, depending on the number of signal paths. This highlights the importance of the number of signal paths in the characterization of wireless devices.
\end{abstract}

Index Terms-Multipath simulator (MPS), signal environment, sparse environment, throughput.

\section{INTRODUCTION}

W IRELESS devices should be characterized under a number of typical propagation channel models in order to ensure a certain minimum performance. A proper definition of channel models has been an interesting research topic in recent years. This letter intends to put some attention on the effect of the exact number of signal paths on high-speed downlink packet access (HSDPA) throughput, when the number of paths is small.

Most of the typical channel models used in industry [1], [2] assume a large number of incident waves at the device under test (DUT). This assumption is fair for many real cases, especially when the user is in an urban environment. However, for other real-life environments, this assumption is debatable, and some studies show that sometimes most of the transferred power is contained in only a few signal paths [3]-[6].

Emulation of multipath environments can be easily performed using mode-stirred chambers (MSCs) [7], which have become one of the established techniques to do over-the-air (OTA) measurements during the last few years. However, as it is shown in [3], the capacity of multiple-input-multiple-output (MIMO) devices is affected when they are working in environments where there is not a large number of signal paths (as rural ones). Therefore, it is necessary,

Manuscript received March 13, 2012; accepted April 22, 2012. Date of publication May 02, 2012; date of current version May 15, 2012. This work was supported in part by MICINN (Project TEC2008-05811) under FPI Doctoral Grant BES-2009-013764.

J. D. Sánchez-Heredia and A. M. Martínez-González are with the Departamento de Tecnologías de la Información y Comunicaciones, Universidad Politécnica de Cartagena, Cartagena 30202 Spain (e-mail: jd.sanchez@upct.es; toni.martinez@upct.es).

P. Hallbjörner is with the SP Technical Research Institute of Sweden, 50115 Borås, Sweden (e-mail: paul.hallbjorner@sp.se).

T. Bolin is with Sony Mobile Communications, 22188 Lund, Sweden (e-mail: thomas.bolin@sonymobile.com).

Digital Object Identifier 10.1109/LAWP.2012.2197591 in order to guarantee a minimum performance for commercial mobile phones, to test these devices in environments where the number of signal waves is limited. This analysis can be easily done using a multipath simulator (MPS) since it allows controlling the number of signal paths [8]-[10].

Taking over the work presented in [11] for passive antennas, this letter presents measurements of HSDPA throughput using an MPS with 2-8 paths. The results are analyzed in terms of average and standard deviation throughput, as well as sensitivity levels, in order to detect whether or not this variable has an important effect over the HSDPA throughput performance of commercial devices.

\section{SETUP}

\section{A. Multipath Simulator}

The MPS used in this study consists of an array of eight dual-polarized antennas (16 antennas in total) distributed in a ring of $1.4 \mathrm{~m}$ radius. Therefore, the MPS simulates a 2-D signal environment, where two signal polarizations are used (vertical and horizontal).

Each of the eight dual antennas is fed through a fiber-optic delay line that introduces a fixed delay, different for each dual antenna. During this study, we only use the dual antennas fed through the four fiber optics with the closest lengths in order to avoid the effect of delays on the study as much as possible. Lengths of the used fibers are 11, 24.5, 81, and $139 \mathrm{~m}$.

Each signal path in the MPS has a phase shifter in order to emulate the fast fading environment experienced in a multipath environment by any device under real use conditions. Attenuators are introduced between fibers and phase shifters to allow switching on and off each of the signal paths via software. Using that feature of the MPS, it is possible to change the number of paths composing the link without changing the DUT position.

\section{B. Performance Metric}

Results are analyzed in terms of HSDPA throughput, measured from the estimation of downlink block error rate (DL BLER). DL BLER is estimated according to the definition in [12]

$$
\begin{aligned}
\text { Throughput }(\%) & =100-\text { DL BLER } \\
\text { DL BLER }(\%) & =\left[\frac{\text { NACK }+ \text { DTX }}{\text { ACK }+ \text { NACK }+ \text { DTX }}\right] \times 100 .
\end{aligned}
$$

Where acknowledgment (ACK) is the percentage of transmission packets that the phone answered after successful cyclic redundancy code (CRC) check, negative acknowledgment (NACK) is the percentage that the phone answered to indicate unsuccessful transmission, and discontinuous transmission 


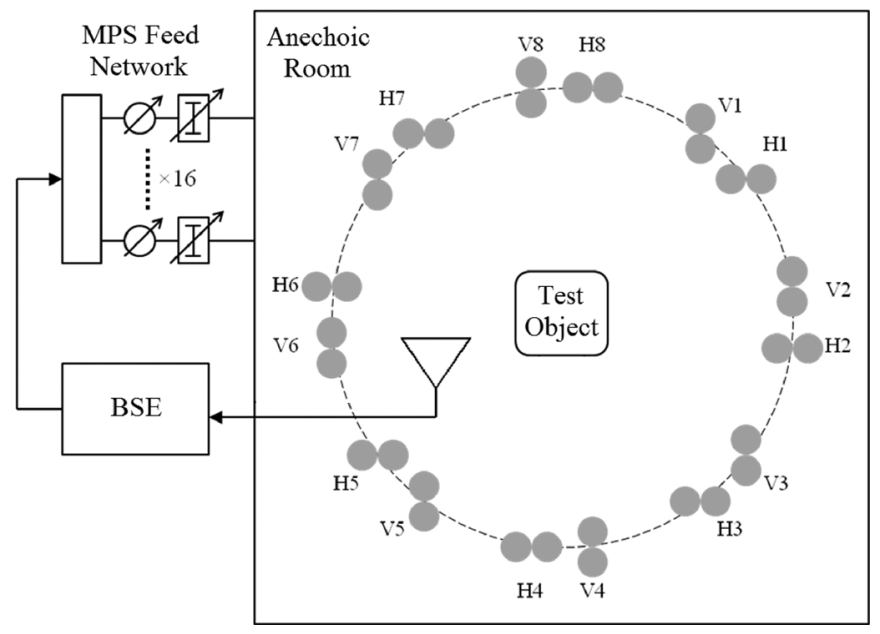

Fig. 1. Block diagram of the setup for HSDPA throughput measurements with an MPS.

(DTX) the proportion that were not answered at all and caused discontinuous transmission.

A Rohde \& Schwarz CMU-200 base station emulator (BSE) is used as measurement equipment. A diagram of the used setup is showed in Fig. 1.

Measurements are performed by doing a sweep over the output power level of the BSE and taking the throughput measurement for each power value. Each of these throughput samples is measured using 2400 HSDPA subframes in about $15 \mathrm{~s}$. A maximum Doppler shift of $50 \mathrm{~Hz}$ is used in the phase shifters to ensure that $15 \mathrm{~s}$ allow enough fading during the measurement.

\section{MEASUREMENTS}

Measurements are performed using a commercial phone HSDPA category-8-capable. Channel 10562 of HSDPA Band I is used (2110 MHz). The HSDPA fixed reference channel (FRC) to be used for these measurements is the H-Set 3, according to the phone HSDPA category [12]. The term "fixed" indicates that both modulation and coding are static during the measurements. 16-QAM modulation is used due its higher sensitivity to fading conditions when compared to QPSK modulation. Other testing conditions are extracted from [13]. Three different cases are studied, with two, four, and eight signal paths.

The MPS comprises 16 antennas, so there are different combinations of antennas that can be used to perform this study. Twelve realizations are taken for each case, using randomly chosen combinations of the antennas. The power is the same in each path in all measurements, so different numbers of paths lead to a different power level at the DUT. This effect is taken into account during the calibration of the MPS, so the BSE is properly configured with the correct link losses. Therefore, differences in performance are not expected to be due to changes in power level.

As 12 randomized combinations of the 16-ring distributed antennas are used (including eight vertical and eight horizontal polarized), there is no preference given to any polarization or angle of arrival (AoA) in order to make the study valid for a

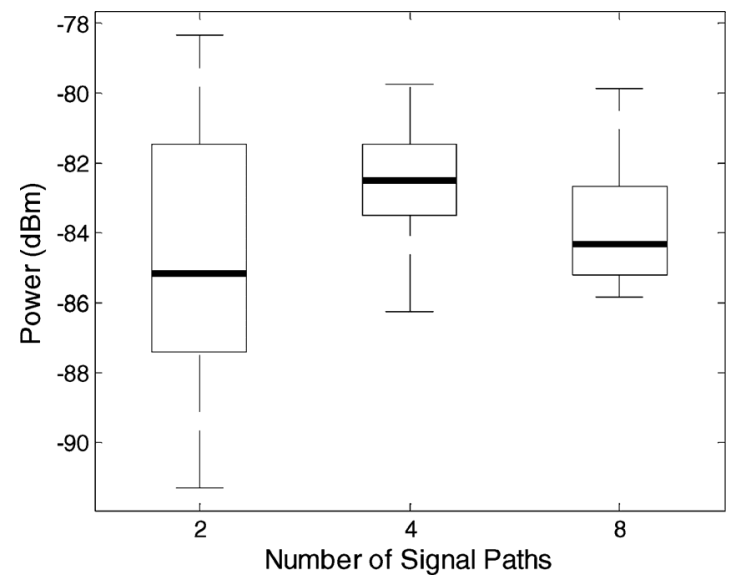

Fig. 2. Power level where $90 \%$ of maximum throughput is reached, as a function of the number of signal paths. Boxes have lines at the lower quartile, median (bold line), and upper quartile values. Whiskers show the extent of the rest of the data.

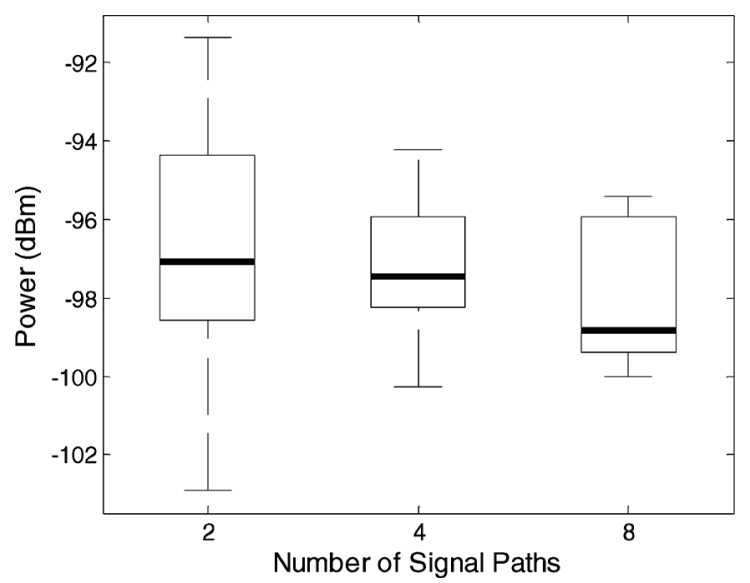

Fig. 3. Power level where $10 \%$ of maximum throughput is reached, as a function of the number of signal paths. Boxes have lines at the lower quartile, median (bold line), and upper quartile values. Whiskers show the extent of the rest of the data.

general case where there is not predominance of any polarization or AoA direction.

The DUT is placed at the center of the MPS antenna array and in free-space condition (without head or hand). Measurements are performed with the phone in vertical position with respect to the plane defined by the antenna array. The antenna radiation pattern in that plane is close to omnidirectional, so the effect of radiation pattern changes is minimized. This effect cannot be completely avoided when using commercial phones.

Fig. 2 is a box-and-whisker plot of the power levels where $90 \%$ of maximum throughput is reached, as a function of the number of signal paths. This $90 \%$ level is commonly used in industry as the sensitivity level of the phone [14]. Fig. 3 shows the results for the power level where $10 \%$ of maximum throughput is reached.

Both the $90 \%$ and the $10 \%$ levels show an important increase in the spread when the number of signal paths is reduced. Differences are especially clear if we compare the case with two signal paths to any of the other cases. The spread with two paths 
TABLE I

Best Case, Median, Mean, and Worst Case at 90\% Throughrut Level FOR DifFERENT Numbers of Signal Paths

\begin{tabular}{|l|c|c|c|}
\hline \hline Parameter & 2 Paths & 4 Paths & 8 Paths \\
\hline Best case 90 \% Level $(\mathrm{dBm})$ & -91.3 & -86.2 & -85.9 \\
Median 90 \% Level (dBm) & -85.1 & -82.5 & -84.4 \\
Mean 90 \% Level (dBm) & -84.7 & -82.7 & -83.8 \\
Worst case 90 \% Level (dBm) & -78.5 & -79.7 & -79.9 \\
\hline \hline
\end{tabular}

TABLE II

Best Case, Median, Mean, and Worst Case at 10\% Throughput LeVel FOR DIFFERENT NUMBERS OF SIGNAL PATHS

\begin{tabular}{|l|c|c|c|}
\hline \hline Parameter & 2 Paths & 4 Paths & 8 Paths \\
\hline Best case 10 \% Level (dBm) & -102.9 & -100.2 & -99.9 \\
Median 10 \% Level (dBm) & -97.1 & -97.4 & -98.9 \\
Mean 10 \% Level (dBm) & -96.8 & -97.3 & -97.9 \\
Worst case 10 \% Level (dBm) & -91.3 & -94.2 & -95.3 \\
\hline
\end{tabular}

is almost twice the spread of the eight-signal-paths case. Even four signal paths show a big difference compared to two.

Measurements are done under fading condition, so there is a spread in the measurement due to the fading. As mentioned before, measurements are taken over a long enough time to ensure that the spread increase due to fading is similar in all cases.

Tables I and II show the best and worst cases, as well as the mean and median values for both the $90 \%$ and $10 \%$ levels. One can observe the different trends of mean and median power values between the $90 \%$ and $10 \%$ power levels. In Table I, it is shown how the trend of the median power values is higher (worst performance) when the number of paths is increased. The effect is the opposite for the case of $10 \%$ throughput level. In that case, an increase in the number of signal paths leads to lower mean values (better performance).

This effect can be explained if we take into account the fact that the HSDPA standard implements a scheme of retransmissions called "redundancy and constellation version coding" [15], which provides an incremental redundancy between retransmissions. When the phone is working at high power (as it is the case of results shown in Fig. 2), no retransmissions are needed, so the phone is more sensitive to the signal fading. On the contrary, when the phone works at low power (results shown in Fig. 3), redundancy is added in the retransmissions, making the phone less sensitive to the signal fading. The signal fading is increased by a greater number of paths. Consequently, performance is reduced at the $90 \%$ level, but improved at the $10 \%$ level, for a larger number of signal paths.

Fig. 4 shows the standard deviation of the measurement for the three cases. Standard deviation is quite similar for the cases with four and eight signal paths, but there is an important increase in the case with two signal paths. It is also seen that standard deviation of HSDPA throughput has an important dependency on the power level.

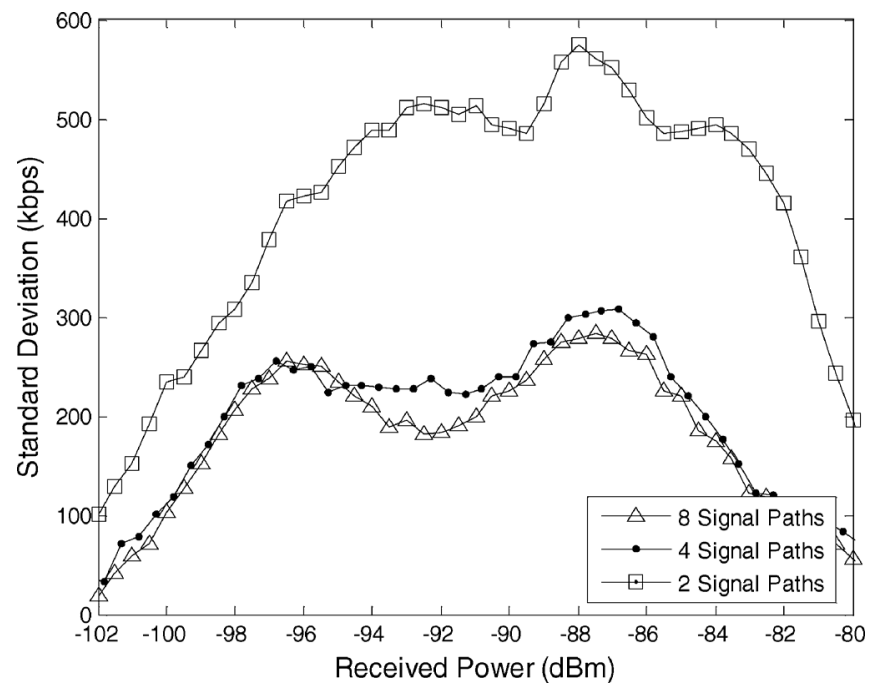

Fig. 4. HSDPA throughput standard deviation as a function of received power.

\section{CONCLUSION}

A commercial mobile phone is characterized in terms of HSDPA throughput using an MPS. The MPS provides a 2-D signal environment, where the number of signal paths can be controlled. Measurements are reported with eight, four, and two signal paths.

The main effect observed when decreasing the number of signal paths is an important increase of the measurement spread. With only two signal paths, differences of more than $10 \mathrm{~dB}$ in the sensitivity level of the phone are shown. These big differences can lead to a mistake differentiating a good and a bad phone.

Apart from that, differences on the mean values are found for different working zones of the DUT. This shows that the number of signal paths has a different effect when the phone is working around the maximum level of throughput ( $90 \%$ level) and when the phone works close to the minimum level ( $10 \%$ level).

Measurements presented in this letter show that the number of signal paths in the channel models used for characterization is important. Most of the already defined channel models assume a large number of signal paths, but some realistic situations could require characterization with a reduced number of signal paths. This characterization can be used to estimate the maximum variations in performance that a user can experience in a real environment with a limited number of signal paths.

\section{REFERENCES}

[1] 3GPP, Sophia-Antipolis, France, "Spatial channel model for multiple input multiple output (MIMO) simulations (Release 10)," Tech. Rep. TR 25.996, 2011 [Online]. Available: http://www.3gpp.org

[2] IST-WINNER, Munich, Germany, "IST-WINNER II deliverable 1.1.2 v.1.2. WINNER II channel models," Tech. Rep., 2007 [Online]. Available: http://www.ist-winner.org/deliverables.html

[3] A. M. Sayeed and V. Raghavan, "Maximizing MIMO capacity in sparse multipath with reconfigurable antenna arrays," IEEE J. Sel. Topics Signal Process., vol. 1, no. 1, pp. 156-166, Jun. 2007.

[4] V. Raghavan, G. Hariharan, and A. M. Sayeed, "Capacity of sparse multipath channels in the ultra-wideband regime," IEEE J. Sel. Topics Signal Process., vol. 1, no. 3, pp. 357-371, Oct. 2007.

[5] X. Lu, X. Chen, G. Sun, D. Jin, N. Ge, and L. Zeng, "UWB-based Wireless Body Area Networks channel modeling and performance evaluation," in Proc. 7th IWCMC, Jul. 4-8, 2011, pp. 1929-1934. 
[6] M. Matthaiou, A. M. Sayeed, and J. A. Nossek, "Sparse multipath MIMO channels: Performance implications based on measurement data," in Proc. 10th IEEE SPAWC, Jun. 21-24, 2009, pp. 364-368.

[7] J. F. Valenzuela-Valdés, A. M. Martínez-González, and D. A. SánchezHernández, "Diversity gain and MIMO capacity for nonisotropic environments using a reverberation chamber," IEEE Antennas Wireless Propag. Lett., vol. 8, pp. 112-115, 2009.

[8] T. Sakata, A. Yamamoto, H. Iwai, K. Ogawa, J.-I. Takada, K. Sakaguchi, and K. Araki, "BER evaluation system for a handset antenna in a multipath environment using a spatial fading emulator," in Proc. ISAP, Seoul, Korea, 2005, pp. 351-354.

[9] L. Rudant, C. Delaveaud, and M. AbouElAnouar, "Synthesizing realistic environments in an anechoic chamber," in Proc. 3rd EuCAP, Berlin, Germany, Mar. 23-27, 2009, pp. 221-225.

[10] P. Hallbjörner, Z. Ying, M. Håkansson, C. Wingqvist, T. Anttila, and J. Welinder, "Multipath simulator for mobile terminal antenna characterization," Microw., Antennas Propag., vol. 4, no. 6, pp. 743-750, 2010
[11] P. Hallbjörner, J. D. Sanchez-Heredia, P. Lindberg, A. M. Martinez Gonzalez, and T. Bolin, "Multipath simulator measurements of handset dual antenna performance with limited number of signal paths," IEEE Trans. Antennas Propag., vol. 60, no. 2, pp. 682-688, Feb. 2012.

[12] 3GPP, Sophia-Antipolis, France, "User equipment (UE) conformance specification; Radio transmission and reception (FDD) (Release 9)," Tech. Rep. TS 34.121, 2010 [Online]. Available: http://www.3gpp.org

[13] 3GPP, Sophia-Antipolis, France, "Measurement of radiated performance for MIMO and multi-antenna reception for HSPA and LTE terminals (Release 10)," Tech. Rep. TR 37.976, 2011 [Online]. Available: http://www.3gpp.org

[14] 3GPP, Sophia-Antipolis, France, "Technical specification group radio access network; User equipment (UE) radio transmission and reception (FDD) (Release 10)," Tech. Rep. TR 25.101, 2011 [Online]. Available: http://www.3gpp.org

[15] 3GPP, Sophia-Antipolis, France, "Technical specification group radio access network; Multiplexing and channel coding (FDD) (Release 10)," Tech. Rep. TS 25.212, 2011 [Online]. Available: http://www.3gpp.org 